\title{
Primary Neural Degeneration in the Guinea Pig Cochlea After Reversible Noise-Induced Threshold Shift
}

\author{
Harrison W. Lin ${ }^{1,3}$, Adam C. Furman ${ }^{3,4}$, Sharon G. Kujawa ${ }^{1,2,3,4}$, and M. Charles Liberman ${ }^{1,3,4}$ \\ ${ }^{1}$ Department of Otology and Laryngology, Harvard Medical School, Boston, MA, USA \\ ${ }^{2}$ Department of Audiology, Massachusetts Eye and Ear Infirmary, Boston, MA, USA \\ ${ }^{3}$ Eaton-Peabody Laboratory, Massachusetts Eye and Ear Infirmary, 243 Charles St, Boston, MA 02114-3096, USA \\ ${ }^{4}$ Program in Speech and Hearing Biosciences and Technology, Harvard-MIT, Cambridge, MA, USA
}

Received: 4 February 2011; Accepted: 1 June 2011; Online publication: 18 June 2011

\begin{abstract}
Recent work in mouse showed that acoustic overexposure can produce a rapid and irreversible loss of cochlear nerve peripheral terminals on inner hair cells (IHCs) and a slow degeneration of spiral ganglion cells, despite full recovery of cochlear thresholds and no loss of inner or outer hair cells (Kujawa and Liberman, J Neurosci 29:14077-14085, 2009). This contrasts with earlier ultrastructural work in guinea pig suggesting that acute noise-induced neural degeneration is followed by full regeneration of cochlear nerve terminals in the IHC area (Puel et al., Neuroreport 9:2109-2114, 1998; Pujol and Puel, Ann N Y Acad Sci 884:249-254, 1999). Here, we show that the same patterns of primary neural degeneration reported for mouse are also seen in the noiseexposed guinea pig, when IHC synapses and cochlear nerve terminals are counted 1 week post-exposure in confocal images from immunostained whole mounts and that the same slow degeneration of spiral ganglion cells occurs despite no loss of IHCs and apparent recovery of cochlear thresholds. The data cast doubt on prior claims that there is significant neural regeneration and synaptogenesis in the adult cochlea and suggest that denervation of the inner hair cell is an important sequela of "reversible" noiseinduced hearing loss, which likely applies to the human ear as well.
\end{abstract}

Correspondence to: M. Charles Liberman - Eaton-Peabody Laboratory Massachusetts Eye and Ear Infirmary $\cdot 243$ Charles St, Boston, MA 02114-3096, USA. e-mail: charles_liberman@meei.harvard.edu
Keywords: acoustic injury, excitotoxicity, inner ear

\section{INTRODUCTION}

The frequency pattern and magnitude of permanent threshold shifts that occur after acoustic injury are well explained by the cochlear pattern and degree of hair cell degeneration and damage to the hair bundles on surviving hair cells (Robertson 1982; Liberman and Dodds 1984). The mechanisms underlying noiseinduced temporary threshold shifts (TTSs) are more difficult to pinpoint, because they likely involve more structures and processes, and because many of the changes may be submicroscopic (Wang et al. 2002).

Despite the complexity of TTS mechanisms, one significant component of the cochlea's acute response to acoustic overstimulation is a type of neuropathy seen as a massive swelling of the auditory nerve terminals in the inner hair cell (IHC) area, when examined within the first 24-48 $\mathrm{h}$ after acoustic overexposure (Spoendlin 1971). Such swelling can be seen after exposures that produce reversible threshold shifts in other animals that are allowed to survive for longer post-exposure periods (Liberman and Mulroy 1982; Robertson 1983), suggesting that neural damage is an important component of TTSs. In guinea pigs, the degree of swelling can be reduced by cochlear perfusion with glutamate antagonists during the noise exposure (Puel et al. 1994) and can be mimicked, in the absence of noise exposure, by cochlear perfusion with the glutamate analog, kainate 
(Pujol et al. 1985), suggesting that the phenomenon reflects a type of glutamate excitotoxicity.

After either kainate perfusion or acoustic overexposure, the terminal swelling disappears within a few days, as the CAP thresholds return to normal (Puel et al. 1998). These observations have suggested there is degeneration, followed by regeneration, of auditory nerve terminals in the adult guinea pig ear. However, until recently, virtually all of the morphological analysis of this afferent neuronal excitotoxicity in the inner ear has been at the ultrastructural level (Puel et al. 1998; Pujol and Puel 1999; Ruel et al. 2007), where quantification is extremely labor-intensive and thus essentially absent from the published record.

Recently, our laboratory has begun to apply immunohistochemistry for pre- and post-synaptic markers (Fig. 1) and high-power confocal microscopy, to quantitatively analyze pathological changes to the afferent innervation in the inner hair cell area (Kujawa and Liberman 2009). This work, in noise-exposed mice, has suggested a very different view of the sequelae of acute noise-induced excitotoxicity, namely (1) that the initial damage to afferent synapses and terminals is widespread throughout the basal half of the cochlea; (2) that there is little or no terminal regeneration or synaptogenesis; (3) that cochlear thresholds, as measured by either auditory brainstem responses (ABRs) or distortion product otoacoustic emissions (DPOAEs), recover despite the loss of $\sim 50 \%$ of the auditory nerve terminals; (4) that amplitudes of gross neural potentials, such as $\mathrm{ABR}$ wave 1, are reduced in proportion to this terminal degeneration; and (5) that the spiral ganglion cells (SGCs) that have lost their unmyelinated peripheral terminals will slowly degenerate over months to years post-exposure.

The present study was designed to address one possible interpretation of this apparent paradox, i.e., that neurons in the mouse ear are uniquely vulnerable to noise. Here, we ask whether the same apparently irreversible neuropathies occur in the guinea pig ear after "reversible" noise-induced threshold shifts, when the morphological and physiological effects of the noise are analyzed quantitatively as in our prior mouse work. The finding that the same primary neural degeneration is indeed present in the noise-exposed guinea pig suggests that this phenomenon is likely common to all mammalian ears and thus a likely covert consequence of repeated noise exposure in the human ear.

\section{MATERIALS AND METHODS}

\section{Animals and groups}

All animals were young female guinea pigs (Hartley strain), approximately $300 \mathrm{~g}$ at time of entry into the

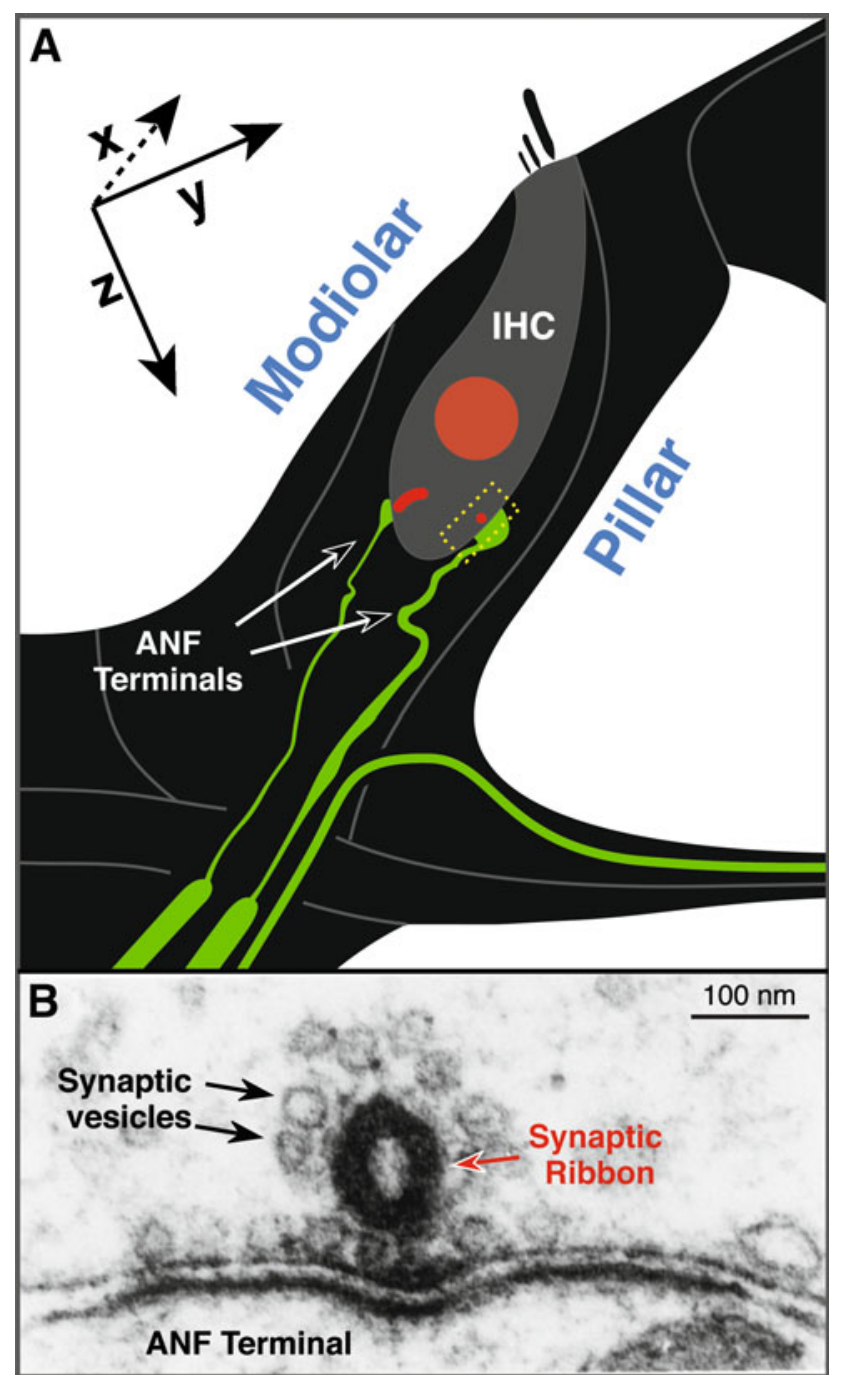

FIG. 1. The normal afferent innervation of the inner hair cell (IHC). A Schematic illustrating two of the 15-20 unmyelinated cochlear nerve terminals (green) making synaptic contact with a single IHC. At each synapse, a pre-synaptic ribbon (red) is present within the IHC. When immunostained with anti-CtBP2 (as in Figs. 4 and 6), the IHC nucleus is also stained. The positions of pillar vs. modiolar sides of the IHC are shown. When viewed in the confocal as epithelial whole mounts, the $x-, y$-, and $z$-planes are oriented as shown. B An electron micrograph of a synaptic complex illustrating the pre-synaptic ribbon and its halo of synaptic vesicles within the IHC (modified from Liberman 1980).

protocol. To assure normal cochlear function, each animal was pre-screened by measurement of ABRs and DPOAEs. Only the left ear was tested in each animal. Animals in the acoustic injury group were exposed to damaging noise at least $24 \mathrm{~h}$ after the prescreening test and then re-tested for cochlear function at varying post-exposure survival times from $24 \mathrm{~h}$ to 6 weeks. A subset of animals was allowed to survive, without further physiological testing, for an additional 2 years. After the final test, animals were euthanized and cochleas were extracted and prepared for histological analysis. All procedures were approved by the 
Institutional Animal Care and Use Committee of the Massachusetts Eye and Ear Infirmary.

\section{Noise exposure}

Animals in the noise groups were exposed for $2 \mathrm{~h}$ to an octave-band noise $(4-8 \mathrm{kHz})$ at either 106 or $109 \mathrm{~dB}$ sound pressure level (SPL). Animals were awake and unrestrained in a small cage on a rotating platform in the sound field within a plywood-lined enclosure measuring roughly $4 \mathrm{ft}$ on a side. Sound pressure level, calibrated via a $1 / 4-\mathrm{in}$. condenser microphone prior to placement of the animal, varied by less than $1 \mathrm{~dB}$ within the space through which the cage rotated. The digital noise waveform was computed with an eighth-order Butterworth filter, and the output of the sound card was amplified (Crown Power Amplifier D75A) and delivered via a compression driver (JBL Model $2446 \mathrm{H}$ ) coupled to an exponential horn.

\section{Physiology: ABRs and DPOAEs}

For measurement of ABRs and DPOAEs, animals were anesthetized with Nembutal (25 mg/kg i.p.) and a fentanyl/droperidol mixture $(0.2$ and $10 \mathrm{mg} / \mathrm{kg}$, respectively, i.m.). Stimulus generation and data acquisition were driven by a custom software package on LabVIEW platform with digital input-output handled by 16-bit sound cards (National Instruments 6052E). Synthesized acoustic stimuli were fed through custom attenuators to a custom, closed acoustic system consisting of two miniature dynamic earphones for delivering stimuli and an electret condenser microphone (Knowles FG-23329-P07) coupled to a probe tube to monitor ear canal sound pressure. The probe-tube microphone was calibrated in a small coupler with a 1/4-in. condenser microphone positioned where the eardrum would be during physiological testing.

For ABRs, stimuli were 5-ms tone pips $\left(0.5 \mathrm{~ms} \cos ^{2}\right.$ rise-fall) delivered in alternating polarity at $40 / \mathrm{s}$ and presented at six frequencies from 2 to $32 \mathrm{kHz}$. Electrical responses were sampled via Grass needle electrodes at the vertex and pinna with a ground reference near the tail and amplified $10,000 \times$ with a $300-\mathrm{Hz}$ to $3-\mathrm{kHz}$ passband. Responses to as many as 1,052 stimuli were averaged at each sound pressure level, as level was varied in $5 \mathrm{~dB}$ steps from below threshold up to $80 \mathrm{~dB}$ SPL. ABR thresholds were defined, by visual inspection of stacked waveforms, as the lowest SPL at which the wave morphology conformed to a consistent pattern (with peak latencies increasing systematically as SPL is reduced). In most cases, this visual threshold corresponded to the level one step $(5 \mathrm{~dB})$ below that at which the peak-to-peak amplitude first appeared to exceed the noise floor.
For DPOAEs, stimuli were two primary tones $f_{1}$ and $f_{2}\left(f_{2} / f_{1}=1.2\right)$, with $f_{1}$ level always $10 \mathrm{~dB}$ above $f_{2}$ level. Primaries were swept in $5 \mathrm{~dB}$ steps from 20 to $80 \mathrm{~dB}$ SPL (for $f_{2}$ ). The DPOAE at $2 f_{1}-f_{2}$ was extracted from the ear canal sound pressure after both waveform and spectral averaging. Noise floor was defined as the average of six spectral points below and six above, the $2 f_{1}-f_{2}$ point. Threshold was computed by interpolation as the primary level $\left(f_{2}\right)$ required to produce a DPOAE of $0 \mathrm{~dB}$ SPL.

\section{Histological preparation and immunostaining}

Whole mounts. To count synaptic ribbons and cochlear nerve terminals in the inner hair cell area, cochleas were prepared as epithelial whole mounts of the organ of Corti. Animals were perfused intracardially with buffered paraformaldehyde (4\%), and the cochleas were extracted and decalcified in EDTA. After decalcification, the cochlear turns were microdissected into $\sim 11$ pieces and then immunostained for confocal examination of the organ of Corti. Ears were typically double-stained with primary antibodies directed against a synaptic ribbon protein (CtBP2, mouse monoclonal from BD Transduction Labs at 1:200) and neurofilament (NF$\mathrm{H}$, chicken polyclonal from Chemicon at 1:1,000). After primary incubation (overnight at room temperature), the secondary antibodies were added: biotinylated donkey anti-mouse (1:200) followed by streptavidin-coupled Alexafluor $568(1: 1,000)$ and Alexafluor 488-coupled goat anti-chicken $(1: 1,000)$ followed by Alexafluor 488-coupled chicken anti-goat $(1: 1,000)$.

Serial sections. To count spiral ganglion cells in Rosenthal's canal, cochleas were prepared as serial sections. Animals were perfused intracardially with a buffered glutaraldehyde $(2.5 \%)$ and paraformaldehyde (1\%) and postfixed overnight. Cochleas were extracted and stained with $1 \%$ osmium tetroxide in distilled water for $1 \mathrm{~h}$, then decalcified in EDTA for $\sim 3$ days. After dehydration in ethanol and propylene oxide, ears were infiltrated with Araldite resins and placed in small embedding molds. After polymerization of the plastics at $60^{\circ} \mathrm{C}$, serial sections were cut at $20 \mu \mathrm{m}$ on a rotary microtome (Leica RM2255) with a carbide steel blade and mounted on glass slides in Permount.

\section{Morphometry}

Whole Mounts. The cochlear frequency map for each ear was determined using a custom plug-in to Image (http:// www.masseyeandear.org/research/ent/eaton-peabody/ epl-histology-resources/). With this plug-in, a series of arc traced over the heads of the pillars in each dissected piece of the cochlea is used to compute 
relative distances along the spiral. Based on a published cochlear frequency map for the guinea pig, these distances are converted into best frequency (Tsuji and Liberman 1997).

Synaptic ribbons in the inner hair cell area were quantified at six cochlear locations corresponding to half-octave intervals of cochlear frequency. At each location, in each ear, two adjacent confocal z-stacks were obtained. Nine ears were analyzed from each group (control vs. exposed); thus, means and standard errors were computed from the 18 observations at each cochlear region. Z-stacks were imaged using a $\times 100$ objective (N.A. 1.4) at $\times 2$ digital zoom with a $z$-step increment of $0.25 \mu \mathrm{m}$. At this magnification, seven to ten inner hair cells and their entire afferent innervation can be contained within each image stack. Each image stack was then ported for offline image analysis via Amira ${ }^{\circledR}$ (Visage Imaging). Using the "connected components" function in Amira, the volumes and $x, y, z$ locations of all ribbons can be determined by choosing an appropriate criterion (pixel value) to define the 3-D iso-surfaces within which all pixel values exceed the chosen criterion (when IHC nuclei were too brightly stained, they could be removed from the counts using the "volume edit" function). Values for ribbons per IHC were computed by dividing the total ribbon count by the number of IHC nuclei in the stack (including fractions).

The apposition of ribbons and auditory nerve terminals was assessed by re-imaging the $z$-stacks as an array of small thumbnails, each centered on the $x y z$ coordinate of an identified ribbon from the Amira analysis. Using custom software, written in $\mathrm{C}++$, the $x y$ projection of the voxel space within $1 \mu \mathrm{m}$ of the center of each ribbon was computed and displayed in an array of miniature $x y$ projections computed from the full set of ribbons. From this re-imaged thumbnail array, in which each image only shows a restricted cube of 3-D space immediately surrounding each ribbon, the numbers of ribbons with closely associated terminals, and the numbers without, can be quickly assessed.

Serial sections. The frequency map for each sectioned ear was determined by (1) reconstructing the cochlear spiral in 3-D, using Neurolucida ${ }^{\circledR}$ software to determine the $x, y, z$ coordinates of the pillar-head junctions in each half-turn from each section, and then (2) ordering this set of $x, y, z$ points and computing distances along the spiral with custom software. Cochlear distance was converted into cochlear frequency according to a published map (Tsuji and Liberman 1997).

SGC density was computed in selected sections by counting the number of SGC nuclei with nucleoli within a rectangular mask $(90 \times 60 \mu \mathrm{m}$ of actual distance) superimposed on the live image obtained through a high N.A. (1.40) oil-immersion objective under Nomarski optics while focusing up and down throughout the section thickness. Since there are overlapping cells visible at different focal planes, Neurolucida ${ }^{\circledR}$ software was used to superimpose markers on counted cells. Cell size was measured by photographing cells at a final magnification of $\times 4,500$ with the same optics used for cell counts. For each cell chosen (20 per section), the focus was set to maximize nuclear area, and measurements were made in ImageJ.

\section{RESULTS}

\section{Physiological analysis}

To assess primary noise-induced neural degeneration in guinea pig, we first varied the intensity of a 2-h exposure to an octave-band noise $(4-8 \mathrm{kHz})$ to find a sound pressure level that produced a severe, but still reversible, noise-induced threshold shift. The degree of acute noise-induced temporary threshold shifts was measured via ABRs and DPOAEs obtained 1 day before, and 1 day after, the exposure. As expected from cochlear mechanical non-linearities (Ruggero 1992), temporary threshold shifts after exposure to $106 \mathrm{~dB}$ SPL were greater at frequencies above the noise band (where the basilar vibration is probably maximal) than within, or below, it (Fig. 2). Temporary threshold shifts were also significantly greater as measured by ABRs (peaking at $\sim 50 \mathrm{~dB}$ - Fig. $2 \mathrm{~A}, \mathrm{C}$ ) than by DPOAEs (peaking at $\sim 25 \mathrm{~dB}$-Fig. 2B, D). This pattern of loss suggests that a component of the threshold shift is due to OHC dysfunction, with an additional contribution of post-synaptic, i.e., neural, damage.

To assess the completeness of subsequent recovery from the temporary threshold shifts, ABRs and DPOAEs were re-measured at several later post-exposure time points. By 10 days post-exposure to the 106-dB noise band, thresholds had recovered to those seen preexposure, as seen in both ABR- and DPOAE-based measures (open circles in Fig. 2). Another group of animals was exposed to the same 2-h octave band at a slightly higher intensity, i.e., $109 \mathrm{~dB}$ SPL. This exposure level increment led to incomplete threshold recovery (data not shown); thus, the 106-dB, 2-h exposure is within $3 \mathrm{~dB}$ of the boundary between reversibility and irreversibility, at least with respect to the threshold for ABRs and DPOAEs.

Prior reports from our laboratory have shown, in mouse models, that primary noise-induced neural degeneration does not appear to affect ABR thresholds but reliably leads to reduction in the amplitude of the ABR wave 1 (Kujawa and Liberman 2009), which reflects the summed activity of the auditory nerve fibers remaining (Melcher and Kiang 1996). The same response phenotype is observed in our 

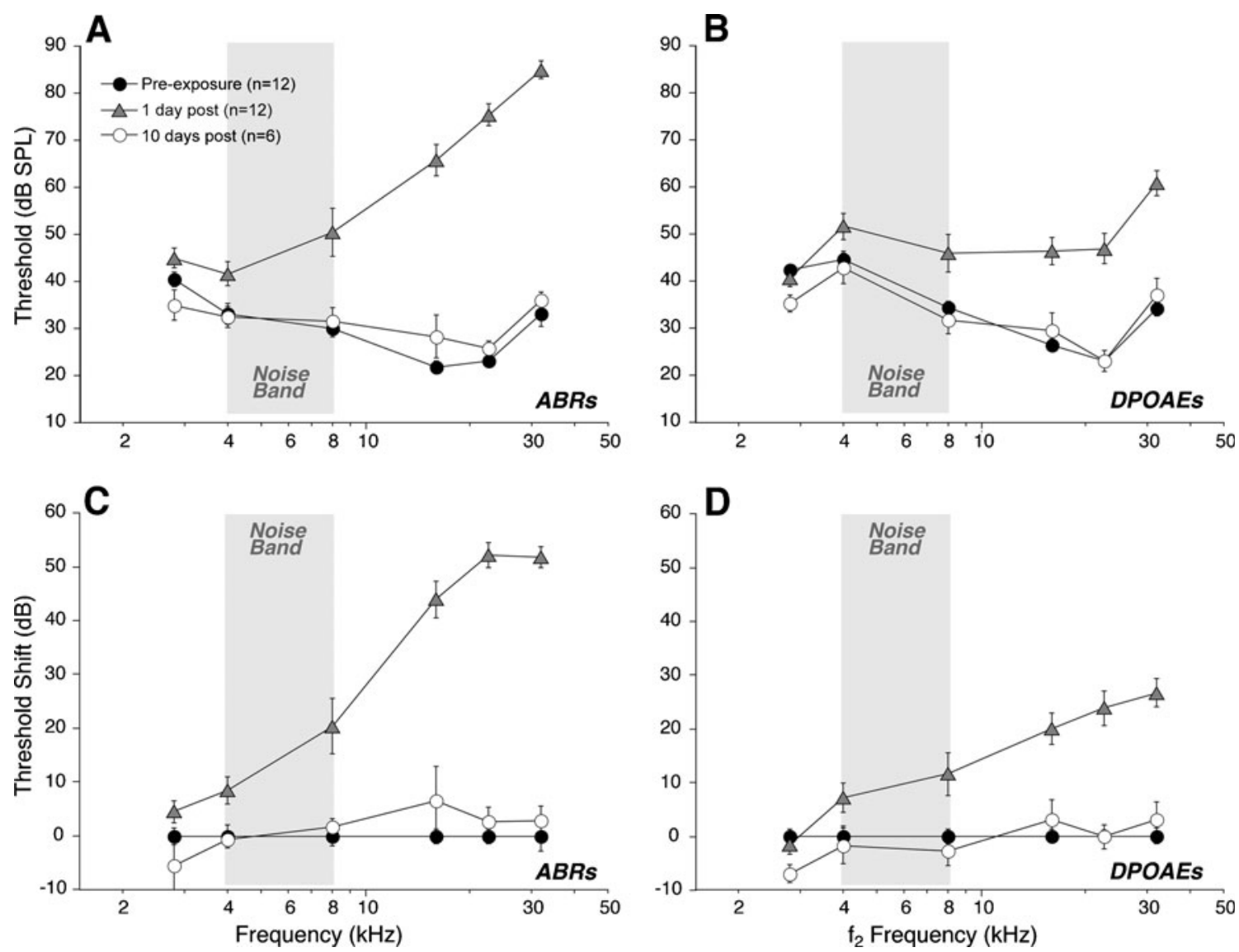

FIG. 2. Despite a large initial threshold shift from exposure to octave-band noise (4-8 kHz) at $106 \mathrm{~dB}$, thresholds recover by 10 days postexposure, as seen via both $\operatorname{ABR}(\mathbf{A}, \mathbf{C})$ and $\operatorname{DPOAE}(\mathbf{B}, \mathbf{D})$ measures. Thresholds shown are group means $( \pm$ SEMs) and are illustrated both as absolute sensitivity $(\mathbf{A}, \mathbf{B})$ and as threshold shift re pre-exposure means $(\mathbf{C}, \mathbf{D})$. Group sizes are shown in the key. Key in $\mathbf{A}$ applies to all panels.

noise-exposed guinea pigs after recovery from "temporary" noise-induced threshold shift. As shown in Figure 3A, the wave 1 amplitudes in the noise-exposed group are, on average, only $\sim 50 \%$ of the control amplitudes at the highest SPL tested $(80 \mathrm{~dB})$, despite complete recovery of the ABR

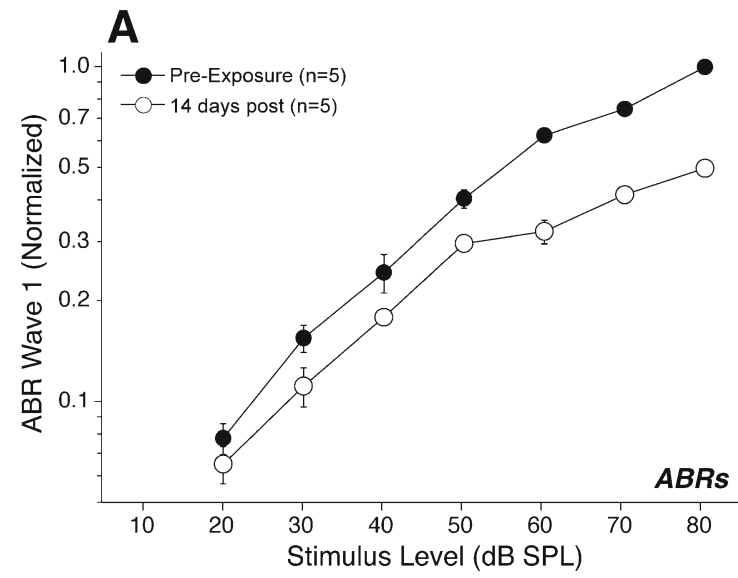

FIG. 3. Although $A B R$ and DPOAE thresholds return to normal after noise exposure, $A B R$ wave 1 amplitude is significantly reduced (A), whereas DPOAE amplitudes recover fully $(\mathbf{B})$. Data are group means $( \pm$ SEMs) for five animals tested at $16 \mathrm{kHz}$ before and 2 weeks after exposure to the $4-8 \mathrm{kHz}$ octave-band noise at $106 \mathrm{~dB}$ for $2 \mathrm{~h}$ (a different thresholds at all test frequencies. In contrast to the reduction in neural suprathreshold response amplitudes, the DPOAE suprathreshold measures (Fig. 3B) return to pre-exposure values, suggesting functional recovery of at least the outer hair cells. The slight enhancement of post-exposure mean DPOAEs, sug-

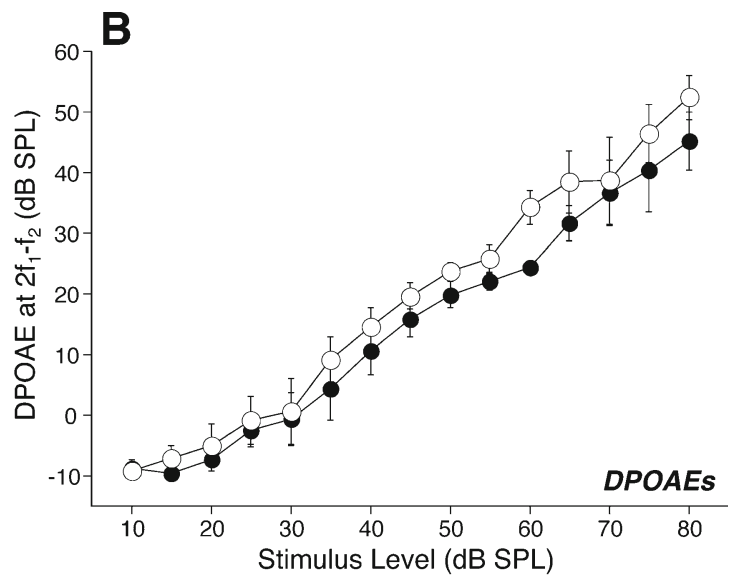

group of animals than those shown in Fig. 2). A For each animal, the wave 1 amplitudes (baseline to peak) are normalized, i.e., expressed as a fraction of the pre-exposure amplitude measured in response to $80 \mathrm{~dB}$ tones. B DPOAE amplitudes are shown as absolute sound pressure levels. Stimulus level refers to $f_{2} ; f_{1}$ level is always $10 \mathrm{~dB}$ greater. 
gested by the data of Figure 3B, has been reported before in noise-exposed mice (Yoshida and Liberman 2000). However, in the present experiment, the difference was not significant by a two-way ANOVA $(p=0.327)$.

\section{Morphometric analysis}

Synaptic ribbons and cochlear nerve terminals. In the mammalian inner ear, most auditory nerve fibers contact a single IHC via a single terminal swelling, at which a single synaptic complex is found, consisting of one pre- synaptic ribbon surrounded by a halo of synaptic vesicles (Fig. 1; Spoendlin 1969; Liberman 1980). Thus, at the light microscopic level, counts of pre-synaptic ribbons can be an informative measure of the afferent innervation in the IHC area of a normal ear. As in previous studies (Kujawa and Liberman 2009), we use antibodies to $\mathrm{CtBP2}$, a major component of the synaptic ribbon (Schmitz 2009), to immunolabel synapses. As shown in the epithelial whole mounts imaged in Figure 4A, anti-CtBP2 labeling in the normal ear reveals small puncta arrayed around the basolateral surface of
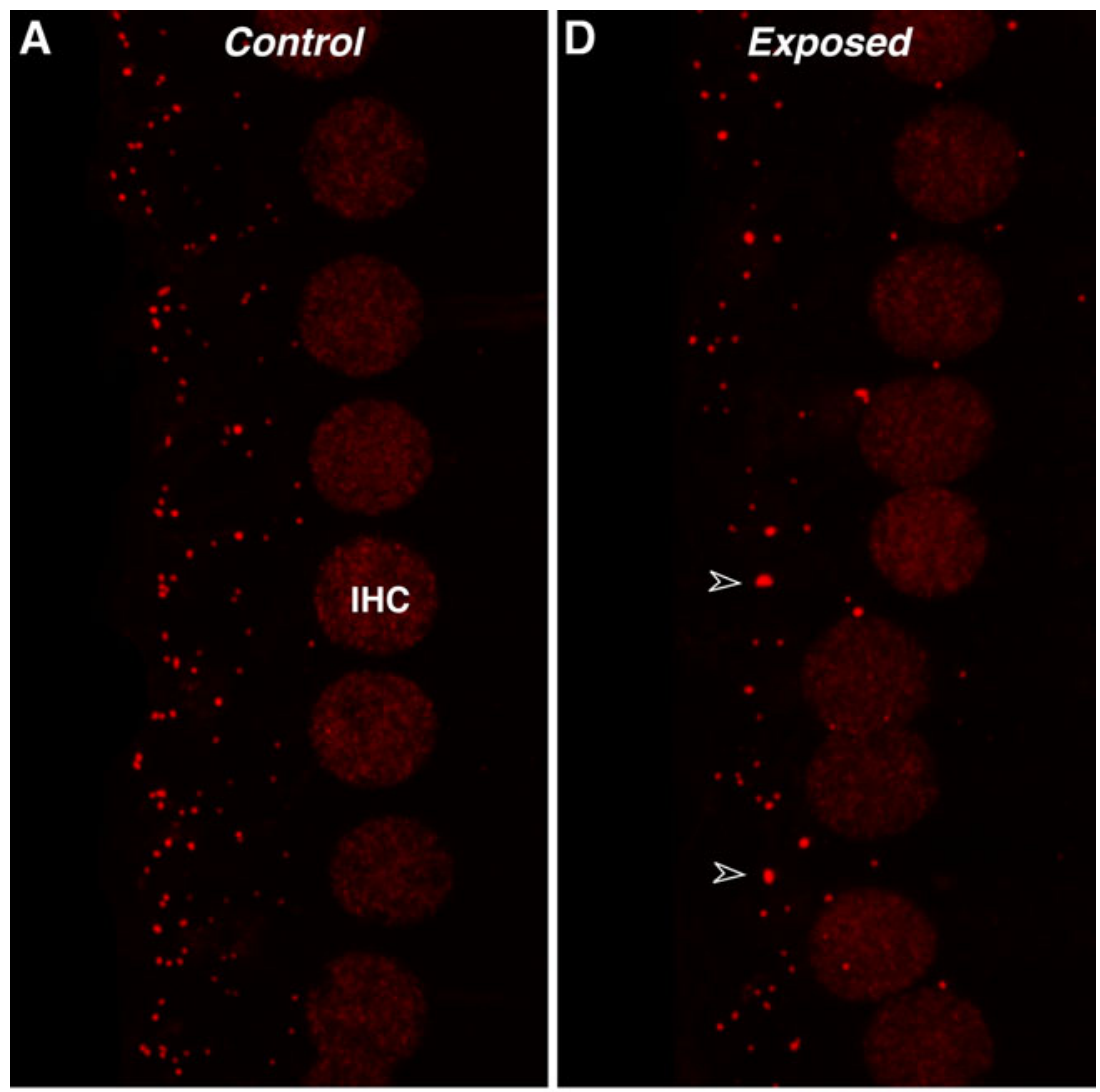

FIG. 4. Synaptic degeneration in noiseexposed ears is seen as loss, disorganization, and dysmorphology of synaptic ribbons. Confocal images of the IHC area in control (A-C) and noise-exposed (D-F) ears immunostained for synaptic ribbons (anti-CtBP2, red) and ANF terminals (anti$\mathrm{NF}$, green; $\mathbf{C}, \mathbf{F}$ only). The exposed ear was harvested 10 days after the $106-\mathrm{dB}$ octave-band noise. For both ears, images were obtained from the $22-\mathrm{kHz}$ region of the cochlea. A, D Maximum projections in the $x-y$-plane (see Fig. 1) from image stacks focused through the entire synaptic regions of seven to eight adjacent IHCs: Their nuclei stain faintly with anti-CtBP2. Arrowheads in D indicate abnormally large ribbons in the exposed ear. B, E The image stacks from $\mathbf{A}$ and $\mathbf{D}$ are rotated to view as $y-z$ projections, i.e. as a cross section through the sensory epithelium (Fig. 1). A dotted line in B divides "modiolar" and "pillar" sides of the IHCs. C, F Same image stack as B, E, but with the green (anti-NF) channel added. Arrowheads in $\mathbf{F}$ indicate abnormally large CtBP2-positive puncta at circumnuclear locations where ANF terminals are not found. Scale bar in $\mathbf{C}$ applies to all panels.
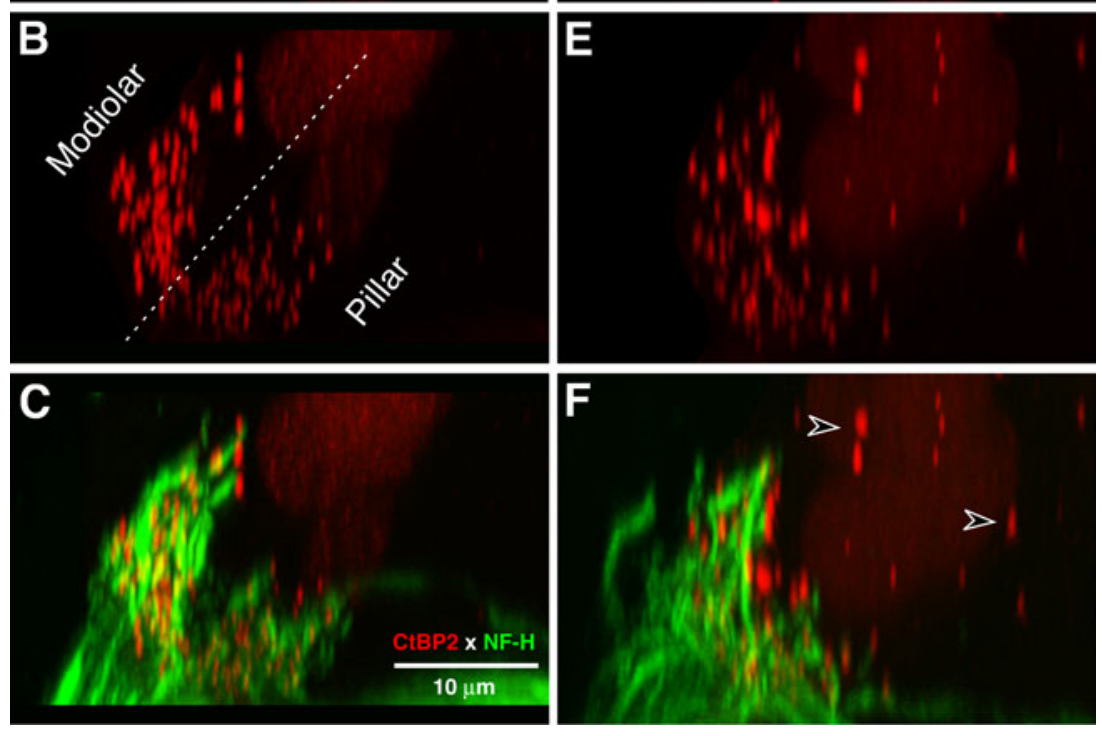
the IHC, in the subnuclear area, as expected based on electron microscopic studies. Counts of these CtBP2positive puncta in normal ears yield an average of $\sim 20$ per IHC in the middle of the cochlear spiral (Fig. 5A), which fits well with ribbon and terminal counts obtained from the IHC area in guinea pigs from serial section electron microscopy (Hashimoto et al. 1991).

When re-imaging these 3-D confocal image stacks to simulate cross-sectional views through the organ of Corti, the ribbon sizes in the normal ear appear to differ on the modiolar- vs. pillar-facing sides of the IHC (Fig. 4B). A recent confocal study in mouse suggested a similar spatial segregation of ribbon sizes in the IHC area and speculated that this organization may reflect the well-studied segregation of high- vs. low-threshold fibers around the IHC circumference (Liberman et al. 2011).

After recovery from noise exposures, at a time when ABR thresholds have recovered but wave 1 amplitudes have not, the confocal analysis of immunostained whole mounts reveals a significant loss of pre-synaptic ribbons throughout the basal half of the cochlea, i.e., throughout the cochlear region where the acute threshold shifts were extreme, as shown in a representative confocal $z$-stack from the $22-\mathrm{kHz}$ cochlear region (Fig. 4D). The quantitative analysis (Fig. 5) shows that, by 10 days post-exposure, ribbon counts are reduced by up to $55 \%$ throughout much of the basal half of the noise-exposed cochleas.

Since the CtBP2 immunolabel also faintly stains the IHC nuclei, it can be seen that there is no loss of IHCs (compare Fig. 4A, D). Brightfield imaging of these whole mounts with Nomarski optics clearly shows hair cell morphology, including hair bundles, confirming the lack of IHC or OHC loss in these ears.

The confocal analysis of noise-exposed ears (Fig. 4A vs. D) also suggests that some remaining ribbons are larger than normal and that some are

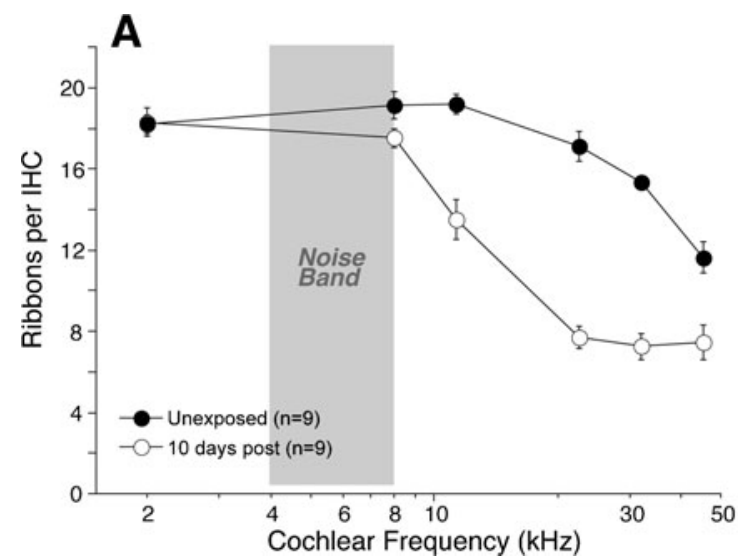

FIG. 5. Quantification of confocal images shows significant loss of synaptic ribbons in the IHC area throughout much of the basal turn in noise-exposed ears. The numbers of ribbons per IHC were counted from confocal $z$-stacks such as those illustrated in Figure 4. Data are group displaced to perinuclear positions where they are never found in the normal ear. The apparent migration of ribbons to positions closer to the nucleus is better seen in the re-imaged cross-section view (Fig. 4E). The migrated ribbons may no longer be apposed to the IHC membrane and thus may no longer be involved in synaptic transmission. This notion is supported by the anti-neurofilament staining (green channel-Fig. 4C, F) which reveals all the unmyelinated terminals of auditory nerve fibers contacting the IHCs. In the normal ear, all the ribbons are located near the auditory nerve terminals; in contrast, after noise exposure, the perinuclear ribbons are far from the closest auditory nerve terminals, which do not appear to migrate with them. Some of the neurofilament positive fibers in the IHC area are olivocochlear efferents.

If some of the remaining ribbons in the noiseexposed ears are no longer closely associated with auditory nerve terminals, then the counts of Figure 5, which include all ribbons regardless of intracellular position, must underestimate the degree of functional denervation of IHCs caused by the noise exposure. To clarify this issue, we used custom software that redisplays the voxel space immediately around each ribbon (Fig. 6B, C). Analysis of the resultant arrays of miniature maximum projections (Fig. 6A) suggests that, after noise exposure, there are twice as many ribbons that are no longer in close apposition to nerve terminals.

Thus, the noise-induced loss of functional synapses may be greater than that suggested by loss of ribbons alone. Exactly how much greater is difficult to tell: The fact that $25 \%$ of normal ribbons are $>1 \mu \mathrm{m}$ from neurofilament immunostaining (Fig. 6) suggests that neurofilaments do not always fill the terminal swelling. Indeed, when normal ears are immunostained with a neural membrane marker $(\mathrm{Na} / \mathrm{K}$ ATPase $)$, there is a

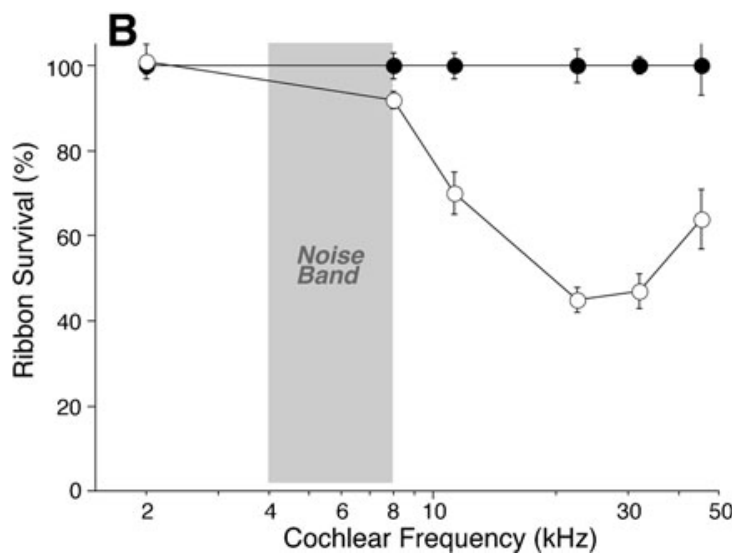

means ( \pm SEMs) and are shown both as absolute counts (A) and as percent survival (B), computed by dividing the exposed-group numbers by the control group data at the same cochlear frequency region. Group sizes are shown in the key. Key in $\mathbf{A}$ also applies to $\mathbf{B}$. 


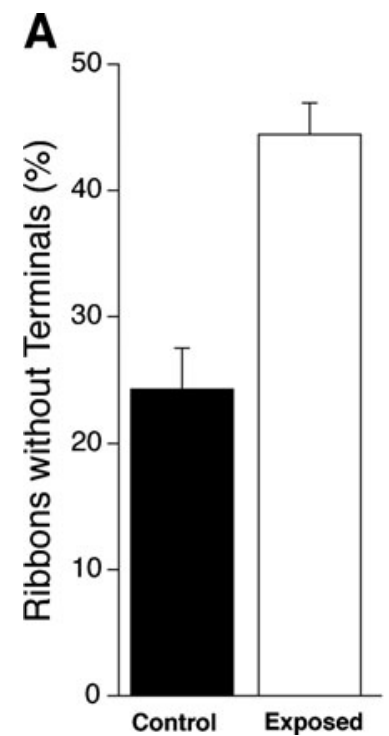

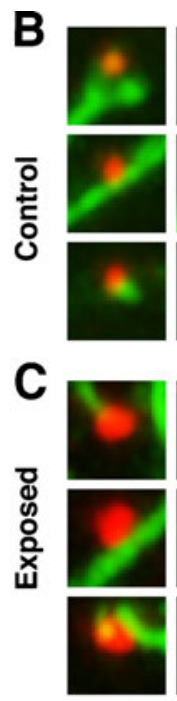

1

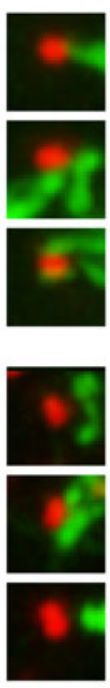

2

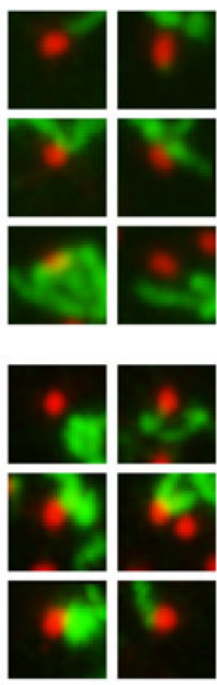

3

FIG. 6. Proximity analysis for ANF terminals and synaptic ribbons suggests that ribbon counts underestimate the synaptic degeneration in noise-exposed ears. A Group means ( \pm SEMs) from ribbon analysis in the 22- and 32-kHz regions show that roughly twice as many ribbons from noise-exposed ears are not closely apposed by ANF terminals.

$100 \%$ correspondence between ribbons and terminal swellings in the IHC area (Liberman et al. 2011).

Spiral ganglion cells. Prior report of primary noiseinduced neural degeneration in mouse showed that, despite a rapid post-exposure loss of synapses and cochlear nerve terminals visible within $24 \mathrm{~h}$ in the organ of Corti, the death of spiral ganglion cells was extremely slow, barely matching the degree of terminal loss by 2 years post-exposure (Kujawa and Liberman 2009). To evaluate whether a similarly slow loss of spiral ganglion cells occurs in the noiseexposed guinea pig, we allowed a small group of animals to survive for 1 year (two ears) or 2 years (four ears) post-exposure, along with a group of agematched controls (four ears at 1 year and two ears at 2 years).

Qualitative analysis of all sections from all ears suggested that (1) there was no loss of ganglion cells in the age-matched controls compared with young normals; (2) there was typically only slight loss of ganglion cells in the noise-exposed ears, even at 2 years post-exposure; and (3) the only obvious signs of reduced ganglion cell density were in the lower basal turn, i.e., in the region corresponding to the maximum ribbon loss (Fig. 5). The micrograph in Figure $7 \mathrm{C}$, from roughly the $22-\mathrm{kHz}$ region in a 2 -year survival ear, shows the most striking reduction of cell density seen in any ear: According to the cell counts in this section, roughly $37.5 \%$ of the cells were surviving. A subtler reduction in cell density is illustrated in Figure $7 \mathrm{~B}$, where counts suggested that $60 \%$ of the cells remained. In other sections from basal turn of noise-exposed ears, the reduc-

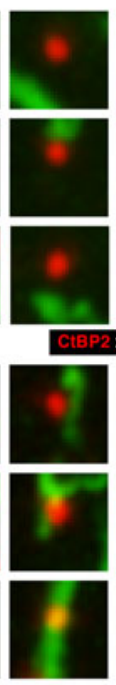

4
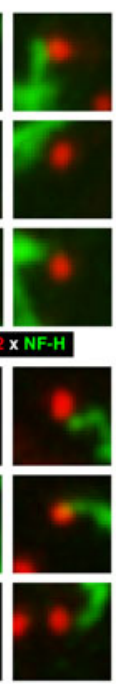

6

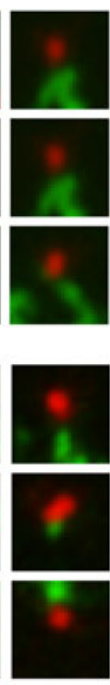

7

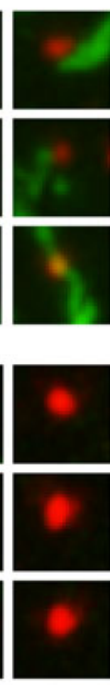

8

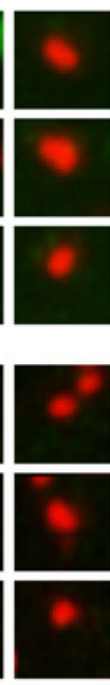

9

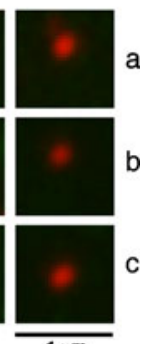

d

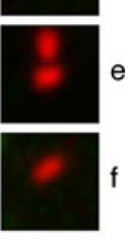

10
Data based on 1,323 ribbons from control ears and 2,432 ribbons from noise-exposed ears. B, C Representative "thumbnail" images on which the analysis in A was based. Each thumbnail shows the $x-y$ projection of the voxel space within $1 \mu \mathrm{m}$ of a ribbon. Ribbons without associated ANF terminals are shown at the right: B 9,10 and $\mathbf{C} 8,9,10$.

tions were minimal. On average, the loss of cells in the 2-year survival ears was less than $25 \%$, even in the most affected region (around $22 \mathrm{kHz}$ : Fig. 7D). Although small in magnitude, the difference in cell counts between control and exposed ears was highly significant $(p<0.01$ by $t$ test). Surviving cells in the $22-\mathrm{kHz}$ region of the exposed ears were also slightly smaller (by $\sim 15 \%$ ) than in control ears (Fig. 7D: $p<0.001$ by $t$ test).

\section{DISCUSSION}

\section{Excitotoxicity in the Cochlea}

Early electron microscopic studies of the acute effects of acoustic overexposure noted widespread and severe swelling of the synaptic terminals of cochlear nerve fibers of ears fixed within 1 to 2 days post-exposure (Spoendlin 1971). Later studies, combining pathophysiology and histopathology, showed that this type of acute neuropathy could be seen even when the exposures caused only temporary threshold elevation, i.e., even if cochlear sensitivity returned to normal in other animals allowed to recover for 1 to 2 weeks after the same exposure (Liberman and Mulroy 1982; Robertson 1983). Morphological studies at both the light and electron microscopic levels have confirmed that noise-induced terminal swelling occurs as part of the acute response to noise in a variety of mammalian ears, including cat (Liberman and Mulroy 1982), guinea pig (Robertson 1983), and mouse (Wang et al. 2002). 

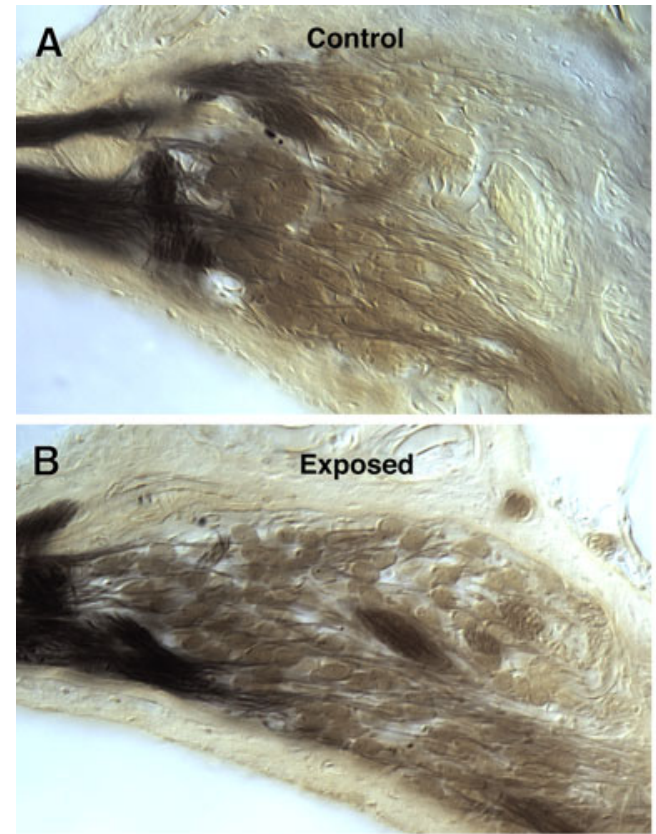

FIG. 7. Two years after noise exposure, there is modest loss of spiral ganglion cells (SGCS) in the basal turn, when compared to age-matched controls. A-C Photomicrographs of cross sections through the spiral ganglion in roughly the $22-\mathrm{kHz}$ region in an age-matched control $(\mathbf{A})$ and in noise-exposed ears from two different animals $(\mathbf{B}, \mathbf{C})$, one with barely detectable degeneration (B) and one with the most severe degeneration

Pharmacological studies of this neuropathic response have suggested that it represents a type of glutamate excitotoxicity. A similar acute swelling of afferent terminals can be elicited by cochlear perfusion with a glutamate agonist (Pujol et al. 1985) without acoustic overexposure, and the degree of noise-induced swelling can be reduced by simultaneous cochlear perfusion with glutamate antagonists (Puel et al. 1998). The observation that targeted deletion of a key gene (GLAST) in the glutamate re-uptake system in supporting cells increases noise-induced threshold shift (Hakuba et al. 2000) is also consistent with the view that glutamate excitotoxicity is an important component of noise-induced cochlear pathology. Whether elicited by noise or drugs, the swelling of cochlear afferent terminals has been seen only in the IHC area. Although recent evidence suggests that glutamatergic transmission also occurs at the outer hair cell's afferent synapse (Weisz et al. 2009), immunostaining suggests expression of different post-synaptic glutamate receptor subtypes at the two classes of afferent terminals (Liberman et al. 2011). These differences could affect $\mathrm{Ca}^{++}$entry and relative susceptibility to excitotoxicity in the inner vs. the outer hair cell areas.

\section{Degeneration vs. regeneration after excitotoxicity}

Electron micrographs of the IHC area of acutely noise-exposed ears often show swollen terminals with
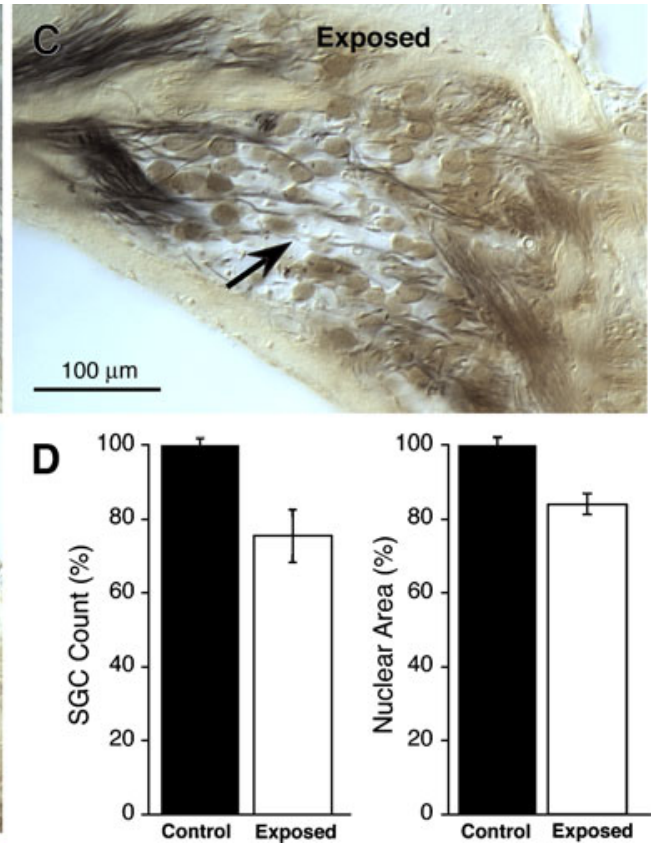

in any of the ears evaluated (arrow in C). Scale bar in C applies to all three micrographs. D Spiral ganglion cell counts and nuclear areas from the $22-\mathrm{kHz}$ region of three age-matched controls (nine sections) vs. three noise-exposed ears (nine sections) with roughly 2-year post-exposure survival (683 days). Means \pm SEM are shown. For both the cell counts and the nuclear areas, data are normalized to the control group means.

ruptured membranes that appear devoid of cytoplasm (Robertson 1983), suggesting absence of synaptic transmission at the time of fixation and irreversible damage if allowed to survive. Although widespread and severe within $24-48 \mathrm{~h}$ post-exposure, excitotoxic swelling is absent from ears evaluated more than 1 week post-exposure. After recovery, normal-appearing synapses can be seen in the IHC area in the electron microscope (Liberman et al. 1986). However, in the absence of quantitative morphological analysis, it is impossible to determine if all terminals recover, or if a subset of the terminals degenerate.

Based on experiments in guinea pig, Pujol and colleagues suggested a third interpretation: that most terminals degenerate and then regenerate within a few $(<5)$ days to re-innervate the IHCs (Puel et al. 1998; Pujol and Puel 1999). The evidence for regeneration includes (1) the observation of growth cone-like structure(s) in electron micrographs of AMPA-treated ears, (2) the presence of normalappearing synapses in the recovered ear despite widespread severe swelling in the acute condition, and (implicitly) (3) the apparent recovery of thresholds for the cochlear compound action potential (Puel et al. 1998; Ruel et al. 2007).

Data from a mouse model of reversible noiseinduced threshold shift suggested a different conclusion about the long-term consequences of acute excitotoxicity. We quantitatively assessed the synaptic 
damage in the IHC area by counting pre-synaptic ribbons and post-synaptic terminals (Kujawa and Liberman 2009) in immunostained cochlear whole mounts. Within 1 day post-exposure, there was $\sim 50 \%$ loss of synaptic ribbons and afferent terminals, despite no loss of inner or outer hair cells. The synaptic loss had not recovered by 8 weeks post-exposure, despite recovery of ABR and DPOAE thresholds by 1 to 2 weeks post-exposure. Loss of spiral ganglion cells eventually mirrored the synaptic degeneration, but the time course was very slow, requiring 2 years before the cell loss approached $50 \%$.

The present study was undertaken, in part, to determine if the discrepancy between these two views arose because the mouse inner ear has a special vulnerability to noise-induced primary neural degeneration. The present results suggest this is not the case. Rather, they suggest that primary neurodegeneration is a fundamental aspect of the ear's response to noise and, therefore, likely true of the human ear as well. By following the same exposure algorithm used in the mouse study, i.e., adjusting the level of a midfrequency noise band to find the most intense 2-h exposure for which the threshold shifts are still fully reversible, we replicated, in guinea pig, all aspects of the neurodegeneration phenotype previously reported in mouse, i.e. (1) immediate and irreversible loss of $\sim 50 \%$ of the IHC afferent synapses, despite no loss of hair cells; (2) corresponding suprathreshold amplitude reductions of roughly $50 \%$ for $\mathrm{ABR}$ wave 1 , despite complete recovery of DPOAE suprathreshold amplitudes; and (3) slowly appearing loss of spiral ganglion cells in the appropriate cochlear locations, despite maintained survival of inner and outer hair cells.

It is also possible that the discrepancy between these two views arises because the ear's response to kainate-induced excitotoxicity, the insult most carefully studied by Pujol and Puel (1999), is fundamentally different from that to noise-induced excitotoxicity: Noise exposure likely has direct effects on both pre- and post-synaptic elements, whereas glutamate receptor agonists such as kainate may only act post-synaptically. In vitro studies suggest that limited reinnervation and synaptogenesis can occur after kainate-induced degeneration of afferent terminals in the neonatal rat cochlea (Wang and Green 2011). Such cochlear explants do not survive in vitro beyond the first post-natal week; thus, the response of the adult inner ear has not been studied in vitro. Since other regenerative capacities, such as the ability to form stem cell spheres, diminish in the cochlear epithelium after the first post-natal weeks (Oshima et al. 2007), the presence of neural regeneration in neonatal ears does not guarantee its maintenance in the adult. The question of whether adult cochlear nerve fibers can spontaneously regenerate peripheral terminals and re-establish afferent synapses with hair cell targets is of fundamental importance to the development of regenerative strategies for the treatment of deafness. The question should be further investigated by quantitative analysis of kainate-treated cochlear epithelia from the adult ear.

\section{Threshold recovery, neural degeneration, and afferent subtypes}

The apparent recovery of thresholds for gross neural potentials such as the ABR wave 1 initially appears paradoxical in the presence of degeneration of at least $50 \%$ of peripheral terminals of cochlear nerve fibers contacting IHCs. However, it has long been known that behavioral thresholds are unaffected by massive loss of cochlear neurons, so long as hair cells are functioning normally (Schuknecht and Woellner 1953). It has also been shown that thresholds of gross cochlear neural responses, such as the compound action potential (CAP) or the ABR, are very insensitive to diffuse loss of type I neurons (or of the IHCs that drive them), so long as the OHC amplifier is functioning normally. For example, in carboplatintreated chinchillas, selective IHC loss of $>50 \%$ was seen in animals with normal CAP thresholds (Liberman et al. 1997). Although sound-evoked cochlear responses are dominated by type I afferent fibers contacting IHCs (Liberman 1982), these gross-potential thresholds are insensitive to diffuse degeneration, because the number of responding type I neurons grows rapidly with increasing sound level, as cochlear vibrations spread along the spiral. More quantitatively, consider that the frequency tuning, or " $\mathrm{Q}$ ", at the $10-\mathrm{kHz}$ place is such that a 5-dB increase in sound level near threshold spreads excitation across an additional $1 \mathrm{kHz}$ of cochlear frequency (Taberner and Liberman 2005). This, in turn, corresponds to about 30 IHCs near $10 \mathrm{kHz}$ in guinea pig (Taberner and Liberman 2005). Even with only ten type I's remaining per IHC (Fig. 5), the summed response of these 300 additional neurons, recruited by a 5 -dB increase in stimulus level, could be as much as $30 \mu \mathrm{V}$, given a contribution to the CAP of $100 \mathrm{nV}$ per fiber (Prijs 1986). Given that a typical "threshold" response criterion for the CAP is $10 \mu \mathrm{V}$ (Liberman et al. 1997), a 5-dB increase in stimulus level should more than compensate for a loss of half the neurons. In other words, there likely is a small underlying threshold elevation $(<5 \mathrm{~dB})$, but we do not see it because level step-size is too coarse and the number of ears is too small with respect to the variance in ABR response amplitudes.

There is another reason why the neural degeneration might be well reflected in amplitude decrements at high sound levels, yet undetectable when expressed as a CAP or ABR threshold. The population 
of type I fibers contacting each hair cell comprises different subtypes, distinguishable on the basis of spontaneous discharge rates and differing in threshold sensitivity (Liberman 1978). As described in Results (Fig. 4C), the clear-cut segregation of ribbon morphologies on the pillar vs. modiolar faces of the normal IHC reflects the segregation of high- and low-threshold fiber subtypes, respectively. If the high-threshold subtypes, i.e., those with low- and medium-spontaneous rates that normally comprise $40 \%$ of the type I fiber group, were selectively lost, as has been suggested in aging gerbil ears (Schmiedt et al. 1996), CAP or ABR thresholds should be unchanged, yet suprathreshold amplitudes should be proportionately decreased. The morphological data obtained in the present study did not reveal a differential pattern of ribbon loss on the two sides of the hair cell; however, ribbon position and ribbon morphologies were also clearly altered by the trauma. Single-fiber recordings will be required to clarify the question of differential vulnerability after noise.

Regardless of which neuronal subtypes are affected, the present results strongly suggest that irreversible neural degeneration is a common result of acoustic overstimulation in the mammalian ear, even if threshold sensitivity returns to normal. Although behavioral thresholds would likely also have returned to normal in our noise-exposed guinea pigs, one might predict there would be difficulties with perceptual tasks in more difficult listening environments, especially if there is selective loss of high-threshold fibers, which are known to be especially resistant to masking by continuous background noise (Costalupes et al. 1984).

\section{ACKNOWLEDGMENTS}

This work was supported by grants from the National Institute on Deafness and other Communication Disorders: R01 DC0188, R01 DC8577, T32 DC0038, and P30 DC5029.

\section{REFERENCES}

Costalupes JA, Young ED, Gibson DJ (1984) Effects of continuous noise backgrounds on rate response of auditory nerve fibers in cat. J Neurophysiol 51:1326-1344

Hakuba N, Koga K, Gyo K, Usami S, Tanaka K (2000) Exacerbation of noise induced hearing loss in mice lacking the glutamate transporter GLAST. J Neurosci 20:8750-8753

Hashimoto S, Kimura RS, Takasaka T (1991) Computer-aided threedimensional reconstruction of the inner hair cells and their nerve endings in the guinea pig cochlea. Acta Otolaryngol 109:228-234

KujaWA SG, Liberman MC (2009) Adding insult to injury: cochlear nerve degeneration after "temporary" noise-induced hearing loss. J Neurosci 29:14077-14085

Liberman MC (1978) Auditory-nerve response from cats raised in a low-noise chamber. J Acoust Soc Am 63:442-455
Liberman MC (1980) Morphological differences among radial afferent fibers in the cat cochlea: an electron-microscopic study of serial sections. Hear Res 3:45-63

Liberman MC (1982) Single-neuron labeling in the cat auditory nerve. Science 216:1239-1241

Liberman MC, Dodds LW (1984) Single-neuron labeling and chronic cochlear pathology. III. Stereocilia damage and alterations of threshold tuning curves. Hear Res 16:55-74

Liberman MC, Mulroy MJ (1982) Acute and chronic effects of acoustic trauma: cochlear pathology and auditory nerve pathophysiology. In: Hamernik RP, Henderson D, Salvi R (eds) New perspectives on noise-induced hearing loss. Raven, New York, pp 105-136

Liberman MC, Dodds LW, Learson DA (1986) Structure-function and correlation in noise-damaged ears: a light and electronmicroscopic study. In: Salvi RJ, Henderson D, Hamernik RP, Colletti V (eds) Basic and applied aspects of noise-induced hearing loss. Plenum, New York, pp 163-177

Liberman MC, Chesney CP, KujaWA SG (1997) Effects of selective inner hair cell loss on DPOAE and CAP in carboplatin-treated chinchillas. Auditory Neuroscience 3:255-268

Liberman LD, Wang H, Liberman MC (2011) Opposing gradients of ribbon size and AMPA receptor expression underlie sensitivity differences among cochlear-nerve/hair-cell synapses. J Neurosci $31: 801-808$

MelChER JR, KIANG NYS (1996) Generators of the brainstem auditory evoked potential in cat. III. Identified cell populations. Hear Res 93:52-71

Oshima K, Grimm CM, Corrales CE, Senn P, Martinez Monedero R, Geleoc GS, Edge A, Holt JR, Heller S (2007) Differential distribution of stem cells in the auditory and vestibular organs of the inner ear. J Assoc Res Otolaryngol 8:18-31

PRIJS VF (1986) Single-unit response at the round window of the guinea pig. Hear Res 21:127-133

Puel JL, Pujol R, Tribillac F, Ladrech S, Eybalin M (1994) Excitatory amino acid antagonists protect cochlear auditory neurons from excitotoxicity. J Comp Neurol 341:241-256

Puel JL, Ruel J, Gervais d' Aldin C, Pujol R (1998) Excitotoxicity and repair of cochlear synapses after noise-trauma induced hearing loss. Neuroreport 9:2109-2114

Pujol R, Puel JL (1999) Excitotoxicity, synaptic repair, and functional recovery in the mammalian cochlea: a review of recent findings. Ann N YAcad Sci 884:249-254

Pujol R, Lenoir M, Robertson D, Eybalin M, Johnstone BM (1985) Kainic acid selectively alters auditory dendrites connected with cochlear inner hair cells. Hear Res 18:145-151

ROBERTSON D (1982) Effects of acoustic trauma on stereocilia structure and spiral ganglion cell tuning properties in the guinea pig cochlea. Hearing Res 7:55-74

ROBERTSON D (1983) Functional significance of dendritic swelling after loud sounds in the guinea pig cochlea. Hearing Res 9:263-278

Ruel J, Wang J, Rebillard G, Eybalin M, Lloyd R, Pujol R, Puel JL (2007) Physiology, pharmacology and plasticity at the inner hair cell synaptic complex. Hear Res 227:19-27

Ruggero MA (1992) Responses to sound of the basilar membrane of the mammalian cochlea. Curr Opin Neurobiol 2:449-456

Schmiedt RA, Mills JH, Boettcher FA (1996) Age-related loss of activity of auditory-nerve fibers. J Neurophysiol 76:2799-2803

Schмiтz F (2009) The making of synaptic ribbons: how they are built and what they do. Neuroscientist 15:611-624

Schuknecht HF, Woellner RC (1953) Hearing losses following partial section of the cochlear nerve. Laryngoscope 63:441-465

SpoEndun H (1969) Innervation patterns in the organ of Corti of the cat. Acta Otolaryngol 67:239-254

Spoendin H (1971) Primary structural changes in the organ of Corti after acoustic overstimulation. Acta Otolaryng 71:166-176

TABERnER AM, Liberman MC (2005) Response properties of single auditory nerve fibers in the mouse. J Neurophysiol 93:557-569 
TsujI J, Liberman MC (1997) Intracellular labeling of auditory nerve fibers in guinea pig: central and peripheral projections. J Comp Neurol 381:188-202

WANG Q, GREen SH (2011) Functional role of neurotrophin-3 in synapse regeneration by spiral ganglion neurons on inner hair cells after excitotoxic trauma in vitro. J Neurosci 31:7938-7949
Wang Y, Hirose K, Liberman MC (2002) Dynamics of noise-induced cellular injury and repair in the mouse cochlea. J Assoc Res Otolaryngol 3:248-268

Weisz C, Glowatzki E, Fuchs P (2009) The postsynaptic function of type II cochlear afferents. Nature 461:1126-1129

Yoshida N, Liberman MC (2000) Sound conditioning reduces noiseinduced permanent threshold shift in mice. Hear Res 148:213-219 